\title{
Identifying cancer-associated fibroblasts as emerging targets for hepatocellular carcinoma
}

\author{
Jie Zhang ${ }^{1 \dagger}$, Chaoyu Gu${ }^{2 \dagger}$, Qianqian Song ${ }^{3 \dagger}$, Mengqi Zhu ${ }^{1}$, Yuqing $X u^{1}$, Mingbing Xiao ${ }^{1 *}$ and Wenjie Zheng ${ }^{1^{*}}$ (D)
}

\begin{abstract}
The tumor microenvironment (TME) is a complex multicellular functional compartment that includes fibroblasts, myofibroblasts, endothelial cells, immune cells, and extracellular matrix (ECM) elements. The microenvironment provides an optimum condition for the initiation, growth, and dissemination of hepatocellular carcinoma (HCC). As one of the critical and abundant components in tumor microenvironment, cancer-associated fibroblasts (CAFs) have been implicated in the progression of HCC. Through secreting various growth factors and cytokines, CAFs contribute to the ECM remodeling, stem features, angiogenesis, immunosuppression, and vasculogenic mimicry (VM), which reinforce the initiation and development of HCC. In order to restrain the CAFs-initiated HCC progression, current strategies include targeting specific markers, engineering CAFs with tumor-suppressive phenotype, depleting CAFs' precursors, and repressing the secretions or downstream signaling. In this review, we update the emerging understanding of CAFs in HCC, with particular emphasis on cellular origin, phenotypes, biological functions and targeted strategies. It provides insights into the targeting CAFs for HCC treatment.
\end{abstract}

Keywords: Cancer-associated fibroblasts, Hepatocellular carcinoma, Tumor microenvironment, Molecular target

\section{Background}

Hepatocellular carcinoma (HCC) is the fourth leading cause of cancer-related mortality worldwide [1]. According to the latest statistics, almost $85 \%$ of $\mathrm{HCC}$ cases occur in developing countries such as Eastern Asia and subSaharan Africa, where chronic hepatitis B virus (HBV) is the most common etiology [2, 3]. In contrast, for developed countries, the primary causes of HCC are hepatitis $\mathrm{C}$ virus (HCV), alcoholic cirrhosis, and non-alcoholic steatohepatitis [4]. Over the past two decades, the incidence of HCC in the USA has increased twofold to threefold, mainly ascribed to the growing HCV-related cirrhosis

\footnotetext{
*Correspondence: zwj007008009@163.com; xmb73@163.com; †Jie Zhang, Chaoyu Gu and Qianqian Song contributed equally to this study

${ }^{1}$ Research Center of Clinical Medicine, Affiliated Hospital of Nantong University, 20 Xisi Road, Nantong 226001, Jiangsu, China

Full list of author information is available at the end of the article
}

and the prevalence of non-alcoholic fatty liver disease (NAFLD) [5, 6]. With the increasing heavy alcohol consumption, obesity, and insulin resistance (IR), more alcohol-related liver disease (ALD)-related HCC and metabolic-related HCC cases have been reported [1, 7].

During the hepatocarcinogenesis, inflammation and fibrosis are well acknowledged as the key drivers. Persistent liver damage can lead to liver fibrosis with the formation of regenerative and dysplastic nodules. Continuous cycles of such destructive-regenerative process eventually gives rise to liver cirrhosis and even carcinogenesis. Indeed, about $1 / 3$ patients with liver cirrhosis will ultimately develop to HCC [8]. However, early diagnosis of $\mathrm{HCC}$ is challenging due to the hidden symptoms. Approximately $80 \%$ of HCC patients are diagnosed at advanced stage, of which the median survival is around 6-8 months. For these patients at advanced stages, treatment strategies include, liver transplantation,

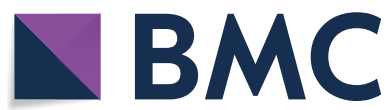

(c) The Author(s) 2020. This article is licensed under a Creative Commons Attribution 4.0 International License, which permits use, sharing, adaptation, distribution and reproduction in any medium or format, as long as you give appropriate credit to the original author(s) and the source, provide a link to the Creative Commons licence, and indicate if changes were made. The images or other third party material in this article are included in the article's Creative Commons licence, unless indicated otherwise in a credit line to the material. If material is not included in the article's Creative Commons licence and your intended use is not permitted by statutory regulation or exceeds the permitted use, you will need to obtain permission directly from the copyright holder. To view a copy of this licence, visit http://creativeco mmons.org/licenses/by/4.0/. The Creative Commons Public Domain Dedication waiver (http://creativecommons.org/publicdomain/ zero/1.0/) applies to the data made available in this article, unless otherwise stated in a credit line to the data. 
topical therapy, and systemic chemotherapy. Additionally, antiangiogenics and immunotherapies are also considered as effective treatment choices. Sorafenib, a small multi-tyrosine kinase inhibitor (TKI), has been approved as the first-line treatment of advanced HCC for over 10 years [9]. Another first line agent, Lenvatinib, has also been evaluated as a novel agent against advanced HCC. For patients that fail in first-line therapy, other antiangiogenic agents (e.g. regorafenib, cabozantinib, and ramucirumab) and immunotherapies (e.g. nivolumab and pembrolizumab) are recommended as second-line strategies [10-14].

Despite the current available treatment strategies, the overall prognosis of $\mathrm{HCC}$ remains unsatisfactory. Therefore, the underlying molecular mechanisms that drive HCC progression and metastasis still need to be investigated. Increasing studies suggest that targeting tumor cells exclusively is insufficient to improve patients' survival, as the malignant behaviors of tumor cells can be greatly modulated by the reconstruction of tumor microenvironment (TME). Given that HCC is often initiated by fibrosis or cirrhosis, the premalignant microenvironment (PME) is considered before tumor formation, which is featured by chronic liver injury, inflammation, and fibrosis in HCC [15]. Once hepatoma occurs, TME, as the suitable environment for tumor cells, takes the place of PME to promote tumor progression [16]. TME in HCC is distinguished by profound ECM remodeling and nontumoral stromal cells, particularly the tumor-associated stromal and immune cells, including cancer-associated fibroblasts (CAFs), B and T cells, neutrophils, endothelial cells, and tumor-associated macrophages (TAMs) [17]. The reciprocal crosstalk among these compartments of TME and HCC cells significantly reinforces proliferation, migration, metastasis and chemoresistance, as well as generation of vasculogenic mimicry (VM) and immunosuppressive induction of neoplastic cells $[18,19]$. As a result, the TME has been identified as a perspective target for developing potential therapeutic agents.

\section{Characterization of CAFs}

CAFs is a heterogeneous group of dynamical fibroblasts that infiltrates in tumor. Distinct from normal fibroblasts, CAFs exhibit a spindle-shaped morphology with large indented nucleus, Golgi complexes, and endoplasmic reticulum (ER). Thus, these CAFs could acquire enhanced metabolic activities and intensive secretory performance [20]. Structurally and functionally, CAFs fertilize the microenvironment for tumor progression by secreting various factors, including ECM proteins (e.g. collagen type-I and ectodysplasin-A), epidermal growth factor (EGF)/fibroblast growth factor (FGF) family members, pro-angiogenesis factors (e.g. hypoxic inducible factor (HIF) and platelet derive growth factor (PDGF)), chemokines (e.g. CXCL and CXCR family members), cytokines (e.g. transforming growth factor- $\beta$ (TGF- $\beta$ )), and different enzymes [e.g. metalloproteinases (MMPs)] [21].

The heterogeneity of CAFs is contributed by the multiple cellular precursors, such as tissue-resident quiescent fibroblasts, bone marrow-derived cells, adipocytes, pericytes and endothelial cells derived from local stromal cells with endothelial-to-mesenchymal transition (EndMT), and cancer cells undergoing epithelial-mesenchymal transition (EMT) [22]. Multiple protein markers have been reported to identify CAFs, including $\alpha$-smooth muscle actin ( $\alpha$-SMA), fibroblast activation protein (FAP), fibroblast specific protein 1 (FSP1 or S100A4), Vimentin, and PDGF receptor (PDGFR)- $\alpha$ and $\beta$ [23]. By contrast, specific markers for distinguishing the heterogeneous CAFs are still lacking. The most acknowledged marker $\alpha$-SMA can be used to identify myofibroblast-like CAFs, vascular muscular cells, and pericytes [24, 25]. Additionally, FAP seems more specific for fibroblasts, though it is also overexpressed in a subset of CD45+ immune cells [26].

The heterogenous CAF subsets have been characterized in different tumors with distinct functions. Recent study demonstrated the existence of four CAF subsets (S1-S4) in breast cancer by concomitant analysis of six markers (CD29, FSP1, FAP, $\alpha$-SMA, PDGFR- $\beta$, and Caveolin1) [27]. Further investigations showed that CAF subset $\mathrm{S} 1$ was associated with immunosuppressive TME by inducing regulatory $\mathrm{T}$ cells (T-regs) differentiation, whereas CAF subset $\mathrm{S} 4$ improved CD8+ T cells infiltration. In addition, four subtypes with specific phenotypic features of CAFs (A-D) were identified in pancreatic ductal adenocarcinoma (PDAC), of which subtype A could furthest enhance proliferation and chemoresistance of cancer cells [28]. Interestingly, in another study of PDAC, CAFs were stratified into two subtypes, i.e. $\alpha-\operatorname{SMA}(+)$ CAFs were distributed around neoplastic cells, while $\alpha$-SMA(-) CAFs localized distantly from cancer cells with stronger paracrine capability of proinflammatory cytokines [24].

Recently, the subtypes and biological functions of CAF are analyzed precisely by using more advanced technologies, such as single-cell sequencing and flow cytometry [29]. Some researchers isolated CAFs cells from fresh HCC tumor tissues. These CAFs are featured by the fibroblastic morphology and activated myofibroblast phenotype with FSP-1 and FAP expression [30]. Moreover, techniques that provide spatial resolution, e.g. multiplexed nucleic acid in situ hybridization and highly multiplexed antibody-based staining, are also utilized to determine whether specific CAF subtype is affected by 
its spatial location within tumor [31]. Recent studies have shown that the precise location may impact the CAFs subtypes with phenotypic discrepancy. For instance, CAF subtype A characterized by periostin was mainly observed at the invasive edge of primary lung tumor, crucial for the tumor capsule formation and metastasis niche. The CAF subtype B marked by myosin-11, commonly identified at the invasive front of tumor, was related to lymph node metastasis and unfavorable prognosis. Besides, podoplanin-marked CAF subtype $\mathrm{C}$ was distributed in the central area of tumor and involved in immunogenic responses [32]. Therefore, identifying subpopulation of CAFs on the ground of the specific role in tumor progression may contribute to innovative targeted therapies and personalized therapies against various cancer types.

\section{CAFs in HCC}

\section{Heterogeneous origins of CAFs in HCC}

As a remarkable feature of CAFs, heterogeneity is embodied with different origins, locations, phenotypes, and functions of cells. Hepatic CAFs commonly marked with $\alpha$-SMA and FAP are widely distributed in tumor septum, fibrous capsule, and hepatic blood sinusoids. According to recent studies, CAFs in HCC can be originated from multiple cell types, including hepatic stellate cells (HSCs), HCC cells undergoing EMT, mesenchymal stromal cells (MSCs), hepatic sinusoidal endothelial cells (HSECs) undergoing EndMT, and peritumoral tissue fibroblasts (PTFs).

One typical source of hepatic CAFs is HSC, which is one major accelerator in liver fibrosis [33]. Based on the genetic cell fate mapping assays, HSCs are suggested as the dominant precursor of $\alpha$-SMA(+) myofibroblasts in most types of liver diseases [33-35]. Under normal conditions, quiescent HSCs are distributed in the disse space functioning as pericytes to store Vitamin A. However, sustained liver injury leads to the activation of HSCs into myofibroblasts, along with enhanced secretion of ECM, chemokines and cytokines [36]. Through migrating to the tissue repairing site, HSCs modulates hepatic fibrogenesis, sequentially causing liver fibrosis and cirrhosis. Once hepatocarcinogenesis, activated HSCs may act as CAFs and dedicate to the formation of HCC fibrous septa and fibrous capsules.

The transformation of HSCs into CAFs is a complicated process, during which intracellular factors from stromal cells and paracrine stimuli from tumor cells are inductive factors [37]. Tan et al. previously reported that human HSC cell line LX2 could be converted into $\alpha-\operatorname{SMA}(+) / \mathrm{FSP} 1(+)$ CAFs with exposure to tumorderived TGF- $\beta$ in vitro [38]. A recent transgenic model also suggested that the hepatocyte-derived platelet derive growth factor-C (PDGF-C) transformed HSCs into myofibroblast-like cells, which in turn produced cytokines to promote the development of HCC [39]. In addition, Zhou et al. indicated that HCC-derived exosomal miRNA-21 could induce the conversion of HSCs into CAFs via downregulating PTEN and activating PDK1/ AKT signaling pathway, subsequently accelerating tumor growth and angiogenesis by secreting massive proinflammatory cytokines [40].

Another source of hepatic CAFs is supposed to be tumor cells. HCC cells locating near the blood sinusoid commonly present with upregulated $\alpha$-SMA or FAP expression. Existing evidence implicate that CAFs with high aggressiveness are originated from $\mathrm{HCC}$ cells undergoing EMT. Consistently, one typical CAF marker, FAP, is also associated with the EMT phenotypes of HCC cells. Zou et al. indicated that hypoxic condition transformed HCC cells into CAFs-like cells with enhanced FAP expression, in parallel with hypoxia inducible factor $1 \alpha($ HIF- $1 \alpha)$ and classical EMT markers (e.g. E-cadherin, Snail, and TWIST) [41]. Moreover, HCC cells exogenously acquired CAF features with the administration of EMT-related cytokine TGF- $\beta$, characterized by remarkably increased $\alpha$-SMA expression [42].

In addition to HSCs and HCC cells, there are other cell types (MSCs, HSECs, and PTFs) that could be transformed into CAFs. Bhattacharya et al. found that MSCs acquired the CAF-like phenotype, characterized by the increased expression of tenascin-C and CXCL12, after co-culture with HCC cell line (SK-Hep1) [43]. As some CAFs distributed in hepatic blood sinusoids, endothelial cells undergoing EndMT provide another source of CAFs in the HCC microenvironment [44]. Apart from that, PTFs can also be converted into CAFs after exposure to lysophostatidic acid (LPA) secreted by HCC cells [45]. In summary, current studies indicated that CAFs in HCC are derived from various cell types, of which HSCs are considered the principal origin. However, other potential sources of CAFs like bone marrow-derived cells and portal fibroblasts (PFs) remain controversial in HCC.

\section{The activation of CAFs in HCC}

As numerous evidences support the indispensable roles of CAFs in HCC growth and metastasis, the cancer cells also fertilized the proliferation and activation of CAFs as feedback. Once activated by corresponding stimuli from cancer cells or TME, CAF progenitors acquire CAF phenotypes and secrete plentiful factors to reinforce their tumor-supporting activities, thereby completing the positive feedback of CAF-HCC cells loop. Such bi-directional activation between cancer cells and stromal cells is critical to cancer progression. 
Mazzocca et al. demonstrated that LPA secreted from HCC cells reinforced trans-differentiation of PTFs into a CAF-like myofibroblastic phenotype, which in turn facilitated proliferation, migration and invasion of $\mathrm{HCC}$ cells. As mentioned above, PDGF-C provoked HSCs into precursors of CAFs with abundant cytokine secretions, ultimately accelerating the progression of HCC [39]. TGF- $\beta$ secreted by CAFs and HSCs has been unequivocally implicated in the malignant phenotypes of HCC cells. Giannelli et al. illustrated that TGF- $\beta$ signaling advanced HCC progression by both intrinsic and extrinsic pathways from activated CAFs in TME [46]. Consistently, Mazzocca et al. further elucidated that inhibition of TGF- $\beta$ could attenuate CAF proliferation by downregulating connective tissue growth factor (CTGF) levels, leading to a significant reduction of HCC growth and dissemination [47]. Recently, STMN1 was found to mediate the intricate crosstalk between HCC cells and CAFs. When co-cultured with HCC cells, HSCs were endowed with CAFs properties and secrete hepatocyte growth factor (HGF), thereby enhancing STMN1 expression of HCC cells through the MET pathway. In turn, upregulation of STMN1 in HCC cells activated HSCs to acquire the CAFs phenotypes [48]. Besides, HCC cells-secreted tissue inhibitor of metalloproteinase 1 (TIMP-1), an endogenous inhibitor for MMPs, has been reported to accelerate cancer progression by initiating the transformation of liver fibroblasts into CAFs. TIMP1 initiated immortalized fibroblasts into CAF-like phenotypes, characterized by elevated expression of CAF markers and enhanced proliferative activities [49]. Currently, it has been recognized that specific sub-populations of CAFs could facilitate cancer stemness by inducing proliferation of cancer stem cells (CSCs) and de-differentiation of cancer cells in a paracrine-dependent pathway. As feedback, CSCs could in turn release specific factors to maintain the activated state of CAFs [50]. Furthermore, Mano et al. identified bone morphogenetic Protein-4 (BMP4) as one key factor involving in the activation of CAFs [51]. Intriguingly, exogenous BMP4 exerted no direct effects on HCC invasion, instead triggering more cytokine production of CAFs in a paracrine manner.

\section{Biological functions of CAFs in HCC progression}

It is noteworthy that CAFs play critical roles in the HCC progression by both direct and indirect interactions with HCC cells. CAFs have been shown to guide the collective tumor cell migration and invasion. Recent study found that the CAFs migrated through the ECM while dragging tumor cells, which was mediated by heterophilic adhesion involving $\mathrm{N}$-cadherin at the CAF membrane and E-cadherin at the cancer cells [52]. Moreover, CAFs secrete multiple types of ECM proteins, growth factors, cytokines and extracellular vesicles (Table 1). With these secretions, CAFs are implicated in modulating ECM, facilitating new vessels, suppressing the anti-tumor immunity, and enhancing EMT and stem feature of HCC cells, which ultimately benefit for HCC initiation with malignant phenotypes (Fig. 1).

\section{CAFs alter ECM in HCC}

The ECM is a dynamic system consisted of collagens, elastin, fibrin, and proteoglycans undergoing constant remodeling. Normal ECM benefits resident cells by providing structural and biochemical support [69]. Nevertheless, altered ECM is accompanied with tumor initiation and progression [70]. Current studies indicate that CAFs secrete a large number of ECM proteins for accelerating tumor progression via matrix degradation, deposition, and stiffening [71]. In the TME, activated CAFs secrete ECM proteins to reinforce the deposition of fibrillar collagens, thereby giving rise to the ECM stiffening. Santamato et al. reported that through secreting the ECM component laminin-5, activated CAFs stimulated the migration and invasion of HCC cells through the MEK/ERK pathway [72]. In addition to boosting metastasis behaviors, Schrader et al. demonstrated that the strengthened tumor matrix stiffness also improved the proliferation of $\mathrm{HCC}$ cells through the PKB/Akt pathway [73]. Contractility is considered as another physical function of CAFs involved in ECM restructuring. By exerting a mechanical force either widening the pores in the ECM or aligning collagen fibers, CAFs could "create the path" for cancer cells to migrate and "guide" cancer cells for directional migration [71]. Following the alterations of ECM, CAFs re-establish a favorable stromal environment for cancer cell invasion. Meanwhile, the matrix answers back by activating CAFs to differentiation. This feedback loop is of great significance in maintaining the activated state of CAFs and constructing the tumorigenicity stroma.

\section{CAFs enhance the stemness of HCC cells}

CSC is one specific subset of cancer cells identified in HCC tissues, maintaining a highly adaptable and dynamic state [74]. Manifested in capabilities of proliferation, self-renewal and migration, CSCs facilitate the initiation, growth, and metastasis of HCC. Under specific conditions, CSC characteristics can be triggered by stimuli from TME [75]. Prior studies have noted that the importance of paracrine pathways in the CAFs-induced stem properties of HCC cells. Lau et al. elucidated that CAFs-derived HGF expanded liver tumor-initiating cells by regulating c-Met/FRA1/HEY1/ ERK cascade $[55,56]$. Focusing on CD24 + HCC cells, 
Table 1 Effects of CAFs-secreted factors on HCC

\begin{tabular}{|c|c|c|c|c|}
\hline CAFs secretion & Molecules & Effect on HCC & Mechanism & References \\
\hline \multirow[t]{3}{*}{ ECM proteins } & BMP4 & Invasion & Trigger cytokines through SMAD pathway & {$[51]$} \\
\hline & COMP & Proliferation, migration, invasion, & Promote EMT process & {$[30]$} \\
\hline & MMP9 & Invasion, metastasis & - & {$[53]$} \\
\hline \multirow[t]{6}{*}{ Growth factors } & HGF & Proliferation & - & {$[54]$} \\
\hline & & Stemness & Facilitate c-Met/ FRA1/ HEY1 signaling and STAT3 signaling & {$[55,56]$} \\
\hline & TGF- $\beta$ & Induce VM & VE-cadherin/MMP2/LAMC2 networks & {$[57]$} \\
\hline & VEGF & Angiogenesis & - & {$[58]$} \\
\hline & PDGF-C & Angiogenesis & - & {$[59]$} \\
\hline & PGF & Angiogenesis & - & {$[60]$} \\
\hline \multirow[t]{9}{*}{ Cytokines } & CXCL12 & Proliferation, migration, invasion & - & {$[49]$} \\
\hline & & Induce VM & Activate VE-cadherin/ MMP2/LAMC2 network & {$[57]$} \\
\hline & & Immunosuppression & $\begin{array}{l}\text { Recruit monocytes and facilitate differentiation into MDSCs } \\
\text { in IL-6-STAT3-dependent manner }\end{array}$ & {$[61]$} \\
\hline & $\mathrm{IL}-6$ & Proliferation, migration, invasion & Activate mTOR signaling & {$[62]$} \\
\hline & & Immunosuppression & Activate STAT3 signaling & {$[61,63,64]$} \\
\hline & & Stemness & IL-6/STAT3/notch signaling & {$[56,65]$} \\
\hline & CCL2, CCL5 & Migration & Hh pathway & {$[66]$} \\
\hline & CCL7, CXCL16 & Migration, invasion & TGF- $\beta$ signaling & {$[66]$} \\
\hline & FOXQ1 & Initiation & Trans-activate NDRG1 & {$[67]$} \\
\hline Extracellular vesicles & Exsomal miR-320a & Suppress proliferation, migration & Inhibit the MAPK pathway & {$[68]$} \\
\hline
\end{tabular}

BMP4, bone morphogenetic Protein-4; ECM, extracellular matrix; COMP, cartilage oligomeric matrix protein; MMP, metalloproteinases; EMT, epithelial-mesenchymal transition; HGF, hepatocyte growth factor; TGF- $\beta$, transforming growth factor- $\beta$; VM, vasculogenic mimicry; IL-6, interleukin 6 ; mTOR, mechanistic target of rapamycin kinase; STAT3, activate signal transducer and activator of Janus kinase; LAMC2, Laminin Subunit Gamma 2; VEGF, vascular endothelial growth factor; PDGF-C, platelet derive growth factor-C; PGF, placental growth factor; $C C L 2 / 5 / 7 / 12$, chemokine (C-C motif) ligand 2/5/7/12; CXCL16, chemokine (C-X-C motif) ligand 16; MDSCs,

marrow-derived suppressor cells; Hh, hedgehog; FOXQ1, forkhead box Q1; NDRG1, N-myc downstream-regulated gene 1; MAPK, mitogen-activated protein kinase

Li et al. emphasized that CAFs induced the stemness via HGF- and IL-6-activated STAT3 pathway in vitro and in vivo [56]. Consistently, Xiong et al. also indicated that CAFs-secreted IL-6 promoted the stem properties of HCC cells through STAT3/Notch signaling [65]. According to the latest study, CAFs and activated PTFs were more likely to recruit CSCs and maintain their stemness by producing a series of cytokines, including IL-6, CCL2, CXCL1, CXCL8, SCGF-b, HGF and VEGF [76]. Sun et al. demonstrated that CAFs-derived cartilage oligomeric matrix protein (COMP) endowed HCC cells with stem-like properties, accelerating the invasion and metastasis of HCC cells [30]. Furthermore, CAFs could also activate Notch3/LSD1 signaling and autophagy-related mTOR pathway of HCC cells, subsequently driving their self-renewal in CSCs [77, 78]. Interestingly, there might be a potential positive loop between CSCs and CAFs. Huang et al. discovered that CAFs secreted TGF- $\beta$ to sustain self-renewal of pluripotent stem cells, which conversely maintained CAFs in an active state with higher secretary and proliferate characteristics through the CAF-CSC crosstalk [50]. Collectively, CAFs regulate stem features of HCC cells to foster the development of HCC.

\section{CAFs promote chemoresistance}

Therapy resistance is an undeniable issue for HCC eradication. Though Sorafenib is recommended as preference for systemic therapy, its efficiency is reported only $30 \%$ in HCC patients with acquired resistance within 6 months. Mechanisms accounting for drug resistance are complicated, of which tumor-stromal interactions may alleviate the sensitivity of HCC cells to anti-cancer drugs. As elucidated above, CAFs secret multiple types of cytokines and vesicles, thereby inducing the therapy resistance of $\mathrm{HCC}$ cells. CAFs-derived HGF enhanced the chemoresistant characteristics of CD73+tumor cells against sorafenib or cisplatin by activating the MEK-ERK1/2 pathway [79]. Khawar et al. found that CAFs-HCC cells-mixed spheroids showed enhanced resistance to sorafenib, while TGF- $\beta$ inhibitors further improved drug efficacy [80]. Consistently, Liu et al. noted that conditioned medium of CAFs conferred HCC organoids resistance to anticancer drugs including Sorafenib, Regorafenib and 5-FU in a paracrine signaling-dependent manner [81]. Aronovich et al. demonstrated that CAFs protected tumor cells from doxorubicin-induced cell death through secretion of CXCL12, which enhanced chemoresistance by binding to CXCR4 [82]. Interestingly, CAFs-derived CXCL12 might facilitate cisplatin resistance by activating 


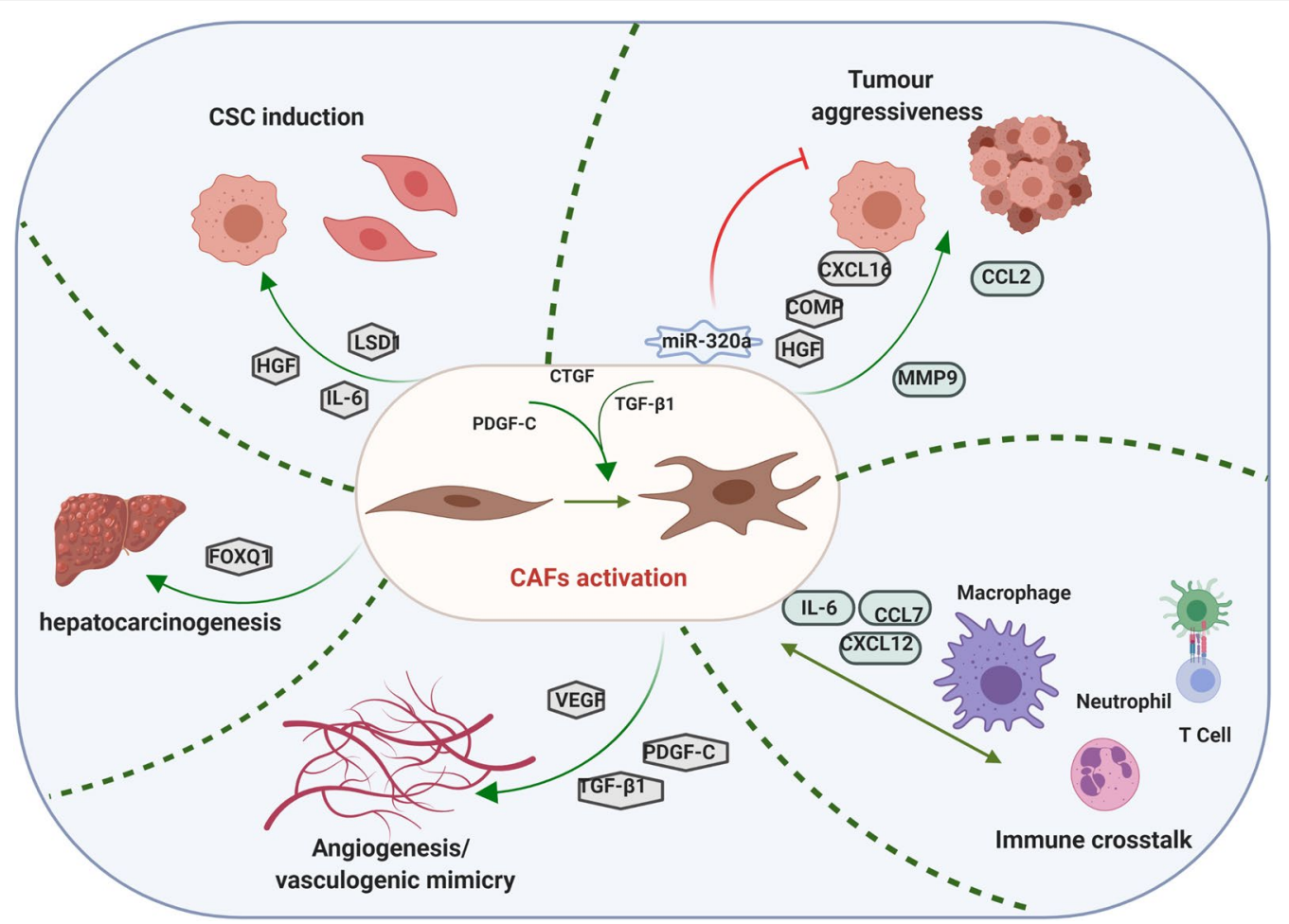

Fig. 1 Effects of cancer-associated fibroblasts on HCC. CAFs could contribute to HCC initiation and progression by various ways, including ECM remodeling, enhancing HCC cells stemness, accelerating angiogenesis and vasculogenic mimicry (VM), as well as inducing immunosuppression. COMP, cartilage oligomeric matrix protein; ECM, extracellular matrix; VM, vasculogenic mimicry; HGF, hypoxic growth factor; PDGF, platelet derive growth factor; EMT, epithelial-mesenchymal transition; PDGFR, platelet-derived growth factor receptor; CTGF, connective tissue growth factor; CSCs, cancer stem cells; VEGF, vascular endothelial growth factor

the Wnt/ $\beta$-catenin pathway [83]. Moreover, Zhang et al. found that neuregulin 1 (NRG1) in CAF supernatant promoted resistance in tumor cells by enhancing HER3 expression [84]. In addition, by analyzing on a threelayered microfluidic tumor-on-a-chip platform, CAFs significantly promoted proliferation and delayed doxorubicin pharmacokinetics of tumor cells when compared to coculture with normal fibroblasts [85]. Furthermore, CAFs-extracellular vesicles (EV)-derived Annexin A6 conferred tumor cells resistance against conventional chemotherapeutics by modulating $\beta 1$ integrin/FAK/YAP signaling [86].

\section{CAFs facilitate angiogenesis and vasculogenic mimicry in HCC}

Tumor angiogenesis refers to the establishment of new blood vessels derived from pre-existing ones. Given that tumor growth and metastasis need nutrients and oxygens, angiogenesis is considered as a hallmark of malignancy, especially for highly-vascularized HCC. Angiogenesis can be regulated by various pro-angiogenic stimulators secreted by cancer cells and stromal cells in the microenvironment. As a major component of tumor stromal, CAFs activated by physiological stimuli like leptin or hypoxia could induce angiogenesis and expedite HCC growth via producing angiogenic factors [87]. Liu et al. indicated that CD90 and placental growth factor (PGF) enriched-CAFs were profoundly correlated with tumor angiogenesis markers (CD31, CD34, and CD105) [60]. Moreover, the overexpression of PGF was correlated with angiogenesis markers and poor prognosis [88, 89]. Notably, the crosstalk between HCC and CAFs contributes to angiogenesis as a positive loop. HCC cells fuel CAFs with elevated secretion of the pro-angiogenesis factor vascular endothelial growth factor (VEGF), which enhances the expansion and tumor vessel formation of HCC cells via upregulating EZH2/VASH1 pathway [90]. These evidences suggest the underlying effects of CAFs on the angiogenesis of HCC in vivo and in vitro.

Extensive studies have suggested anti-angiogenic drug as an effective anti-tumor strategy, which target vascular endothelial cells to block blood supply to tumor cells. However, angiogenesis may not be the exclusive mechanism that tumors acquire microcirculation. Aggressive tumor cells are capable of forming highly patterned vascular channels and vasculogenic mimicry (VM), in which 
tumor cells generate their own blood-delivery channels without the participation of endothelial cells [91]. Of interest, Yang et al. found that CAFs ratio in $\mathrm{VM}+\mathrm{HCC}$ tissue was significantly higher than that in VM- tissue. Further investigation disclosed CAFs isolated from fresh HCC tissues remarkably enhanced VM formation in vitro and in vivo through enhancing the expression of VE-cadherin, MMP2 and laminin5 $\gamma 2$ via TGF- $\beta R 1$ and CXCR4 in $\mathrm{HCC}$ cells, suggesting the roles of CAFs in facilitating VM formation and angiogenesis [57].

\section{CAFs induce immunosuppressive milieu in HCC}

Increasing studies highlight the immunosuppressive functions of TME in favoring the HCC progression and potential drug resistance. The initiation of such immunetolerant microenvironment is mediated by numerous inflammatory factors such as growth factors, cytokines, chemokines generated by multiple cell types co-existed and interacted in TME $[92,93]$. Of them, the key constructive cells include TAMs, marrow-derived suppressor cells (MDSCs), tumor-associated neutrophils, CAFs, as well as infiltrating $\mathrm{T}$ cells and natural killer (NK) cells [94].

Accumulating studies have emphasized the major role of CAFs in shaping the immunosuppressive TME. By producing immune prohibitive cytokines and immune checkpoint ligands, CAFs recruit immunosuppressive cells like MDSCs and peripheral blood neutrophils, and modulate immune cells differentiation such as monocytes and dendritic cells (DCs) [95]. MDSCs are heterogeneous populations composed of immature myeloid cells with various differentiation states. With the capability to inhibit the proliferation and activity of $\mathrm{T}$ and NK cells, MDSCs restrain the immune response of the TME. In a murine model of hepatic cancer, Yang et al. found that $\mathrm{FAP}(+) \mathrm{CAFs}$-secreted CCL2 recruited circulating MDSCs with high CCL2R expression via STAT3CCL2 signaling. Conversely, neutralizing anti-CCL2 antibody or knockdown of CCL2 remarkably blocked the migration of MDSCs and abrogated FAP $(+)$ CAFmediated tumor promotion [96]. In addition, Cheng et al. indicated that CAF-derived IL- 6 could recruit and regulate the survival, activation, and function of neutrophils through STAT3-programmed cell death ligand 1 (STAT3-PDL1) signaling cascade, afterward contributing to the immune tolerance of HCC cells [64]. According to the study of Li et al., CAFs produced IL-6 could activate STAT3 in DCs, boosting the generation of regulatory DCs, which are characterized by tolerogenic phenotypes with high expression of suppressive cytokines. Moreover, these CAF-educated DCs could confer T cells a suppressive phenotype with the decreased production of IFN $-\gamma$ in CD8 $+\mathrm{T}$ cells and the expansion of
CD4+CD25+Foxp3+ Tregs expansion [97]. Apart from that, hepatic CAFs could also recruit monocytes by stromal cell-derived factor (SDF)-1a/CXCR4 pathway, and stimulate the monocytes differentiation into MDSCs in IL-6-STAT3-dependent manner, thereby eliminating the anti-tumor immune responses by inhibiting $\mathrm{T}$ cell proliferation and functions [61]. Furthermore, HCC derived CAFs inactivated NK cells by secreting indoleamine 2,3-dioxygenase (IDO) and PGE2, thereby forming an immune-tolerant niche appropriate for $\mathrm{HCC}$ progression [97]. Another study reported that CAFs secreted TGF- $\beta$ could improve the Tregs proliferation, thus accelerating the HCC growth in the hepatic TME [63]. These evidences demonstrated that CAFs contribute to the immunosuppression functions of $\mathrm{HCC}$ via interacting with various types of immune cells in TME.

\section{CAFs associate with clinical outcome of HCC}

The molecular heterogeneity of $\mathrm{HCC}$ is manifested in both tumor and tumor stromal cells, triggering distinct clinical outcomes. As CAFs contribute to HCC progression, this key tumor stromal component has the potential to predict the survival of HCC patients. Current studies have found the correlation of $\alpha$-SMA $(+)$ CAFs with poor clinical outcome in HCC cases. Lau et al. reported that $\alpha$-SMA expression was negatively correlated with disease-free survival (DFS) of HCC patients [55]. Consistent with it, Fang et al. also found HCC cases with higher proportion of a-SMA(+) CAFs had shorter DFS [98]. Another study showed that the HCC recipients with $\alpha$-SMA(+) CAFs had higher risk of recurrence after liver transplantation [99]. Additionally, Yang et al. suggested a-SMA(+) CAFs as a biomarker for HCC progression due to the implications in metastasis and tumor staging [100]. At gene level, the encoding gene of a-SMA, ACTA2, was also associated with clinicopathologic features including TNM stage, tumor size, tumor encapsulation, and vascular invasion. Moreover, HCC patients with higher expression of ACTA2 exhibited shorter over survival (OS) and higher recurrent rate [67].

It was recently reported that CAFs-expressed CD90 was associated with clinicopathologic features of HCC. Zhao et al. pointed out that high levels of CD90(+) CAFs were correlated with advanced pathological grade, satellite lesion, PVTT, and HCC recurrence. Furthermore, CD90(+) CAFs were indicative of unfavorable outcome of HCC patients after hepatic resection [101]. Other than cell surface markers, CAFs derivations are candidate predictors for HCC prognosis. Liu et al clarified that CAFs-derived PGF, specifically contributing to the neo-angiogenesis, was vastly correlated with unfavorable prognosis of $\mathrm{HCC}$ patients [102]. Consistently, Xu et al. signified that high expression of PGF in peri-tumor 
tissues were indicative of poor OS of HCC patients [89]. In addition, the expression of PGF was also positively associated with early recurrence of HCC patients [88]. Significant telomere attrition has been observed in tumor tissues compared to their normal counterparts. Ma et al. suggested that shortened telomeres in CAFs resulted in decreased survival time and increased recurrence rate of HCC patients. This finding was further confirmed in an independent cohort from The Cancer Genome Atlas (TCGA) public database [103]. Taken together, the surface molecular markers and derivations of CAFs may provide potential prognosis of $\mathrm{HCC}$ outcome.

\section{Target CAFs in HCC}

Given the remarkable tumor-supportive roles, CAFs are evaluated as promising therapeutic targets for cancer intervention. It is generally accepted that cancer cells are liable to develop resistance to various types of therapies due to the genetic instability. Comparatively, CAFs are genetically more stable and less prone to acquire drug resistance [104]. Generally, the strategies of targeting CAFs include targeting specific markers, endowing CAFs with tumor-suppressive phenotype, depleting the CAFs' precursors, and repressing the secretions or downstream signaling molecules of CAFs. Herein, we summarized current studies regarding CAFs-targeted treatment for HCC (Table 2).

\section{Engineer CAFs to acquire tumor-constraining features} Indeed, it is unavoidable to precisely target CAFs without damaging normal cells, which limits the application of marker depletion. Particularly, directly ablating the vital stromal component CAFs may break the homeostasis and inversely exacerbate the disease. Thus, inactivating CAFs into a quiet state or conferring CAFs the tumor suppressor phenotype might be preferable therapeutic

Table 2 Strategies of targeting CAFs in HCC

\begin{tabular}{|c|c|c|c|c|}
\hline Therapeutic approach & Therapeutic setting & Mechanism & Major effects on HCC & References \\
\hline \multirow[t]{3}{*}{ Engineer CAFs } & miR-335 & - & Inhibit proliferation and invasion & [105] \\
\hline & miR-320 & $\begin{array}{l}\text { Target PBX3 to suppress MAPK } \\
\text { pathway }\end{array}$ & Inhibit proliferation and metastasis & {$[106]$} \\
\hline & miR-101 & $\begin{array}{l}\text { Target TGF- } \beta \text { / SDF1-VE-cadherin/ } \\
\text { MMP2/LAMC2 networks }\end{array}$ & $\begin{array}{l}\text { Suppress CAFs-promoted VM } \\
\text { formation }\end{array}$ & [57] \\
\hline \multirow[t]{5}{*}{ Target CAFs' precursors } & Sibrotuzumab & - & Inhibit HSCs activation & [107] \\
\hline & DFOG & $\begin{array}{l}\text { Suppress FOXM1 expression and } \\
\text { HGF secretion }\end{array}$ & $\begin{array}{l}\text { Inhibit CSC features and activation } \\
\text { of HSCs }\end{array}$ & {$[108]$} \\
\hline & Metformin & - & $\begin{array}{l}\text { Inactivate HSCs and abrogate } \\
\text { hepatocarcinogenesis }\end{array}$ & [109] \\
\hline & Curcumin & $\begin{array}{l}\text { Inactivate ROS/ HIF-1a/CTGF } \\
\text { signaling }\end{array}$ & $\begin{array}{l}\text { Suppress HSCs-induced angiogen- } \\
\text { esis and invasion }\end{array}$ & [110] \\
\hline & $\begin{array}{l}\text { a-bromomethylene } \\
\text { phosphonate- LPA }\end{array}$ & & $\begin{array}{l}\text { Block the transformation from PTFs } \\
\text { to CAFs }\end{array}$ & [45] \\
\hline \multirow[t]{5}{*}{$\begin{array}{l}\text { Target paracrine productions of } \\
\text { CAFs }\end{array}$} & IL-6 neutralizing antibody & Inhibit IL-6 signaling & $\begin{array}{l}\text { Deplete stem cell-like properties of } \\
\text { HCC cells }\end{array}$ & {$[65]$} \\
\hline & LY2109761 & $\begin{array}{l}\text { Inhibit the synthesis and release } \\
\text { of CTGF }\end{array}$ & $\begin{array}{l}\text { Reduce HCC growth and dissemi- } \\
\text { nation }\end{array}$ & [47] \\
\hline & CCL2,5,7 antibodies & Inhibit Hh and TGF- $\beta$ pathways & Inhibit tumor migration & {$[66]$} \\
\hline & T0901317 & $\begin{array}{l}\text { Abrogate TGF- } \beta \text {-induced pheno- } \\
\text { types through LXRa interactions }\end{array}$ & Suppress HCC growth & [42] \\
\hline & RvD1 & $\begin{array}{l}\text { Suppress COMP secretiom by } \\
\text { targeting FPR2/ROS/FOXM1 } \\
\text { signaling }\end{array}$ & $\begin{array}{l}\text { Impede CAFs-induced EMT and } \\
\text { stemness features of HCC cells }\end{array}$ & {$[30]$} \\
\hline \multirow{4}{*}{$\begin{array}{l}\text { Target CAFs-mediated signaling } \\
\text { and pathways }\end{array}$} & Dorsomorphin & Inhibit SMAD signaling & Impede the activation of CAFs & [51] \\
\hline & P38 MAPK inhibitor & Block CAF-M-MDSC crosstalk & Provoke antitumor immunity & [111] \\
\hline & Rapamycin & Suppress mTOR-signaling pathway & $\begin{array}{l}\text { Inhibit HCC cells proliferation, } \\
\text { migration and invasion }\end{array}$ & {$[62]$} \\
\hline & Cryptotanshinone & Inactivate p-STAT3 signaling & $\begin{array}{l}\text { Abrogate stem cell-like properties } \\
\text { of HCC cells }\end{array}$ & {$[65]$} \\
\hline
\end{tabular}

COMP, cartilage oligomeric matrix protein; TGF- $\beta$, transforming growth factor- $\beta$; VM, vasculogenic mimicry; IL-6, interleukin 6; mTOR, mechanistic target of rapamycin kinase; STAT3, activate signal transducer and activator of Janus kinase; $C C L 2 / 5 / 7 / 12$, chemokine (C-C motif) ligand 2/5/7/12; CXCL16, chemokine (C-X-C motif) ligand 16; MDSCs, marrow-derived suppressor cells; Hh, hedgehog; FOXQ1, forkhead box Q1; CTGF, connective tissue growth factor; MAPK, mitogen-activated protein kinase; RvD1, Resolvin D1; MMP, metalloproteinases; EMT, epithelial-mesenchymal transition; VM, vasculogenic mimicry; ROS, reactive oxygen species; LPA, lysophostatidic acid; HCC, hepatocellular carcinoma; CAFs, cancer-associated fibroblasts; HSCs, hepatic stellate cells; PTFs, peritumoral tissue fibroblasts 
approaches. In a CCl4- and alcohol induced liver fibrosis model, most activated myofibroblasts were declined into inactivated HSCs during regression of liver fibrosis by removing etiological agent [112]. In addition, researchers have employed nanoparticles that can be uptake by CAFs to genetically modify CAFs in situ, whereby CAFs may be engineered as a tumor depletion center to persistently release anti-tumor cytokines [113]. In xenograft mice models, the engineered CAFs served as a tumor-directed cytotoxic chemotherapeutic reservoir to trigger the apoptosis of neighboring tumor cells [114]. Interestingly, the apoptosis of adjacent tumor cells reciprocally reverts CAFs to a quiescent state, orchestrating the suppressive microenvironment that is favorable for a second-wave of nano-therapy. Thus, engineered CAFs may function as cytotoxic drugs, providing a new paradigm for tumor therapy.

CAFs communicated with cancer cells partly by extracellular vesicles (EVs) in a paracrine way. Several studies indicated that downregulation of the signals carried by CAF-derived EVs or exosomes contributed to the proliferation and metastasis of hepatoma cells. In contrast, overexpressing certain miRNAs may confer CAFs the tumor-suppressive phenotypes, providing potential options to impede HCC progression. Wang et al. reported that downregulation of miR-335 was observed in cancer cells and CAFs, which was beneficial to cancer development. Moreover, miR-335-engineered CAFs acquired the anti-tumor phenotypes against neighboring cancer cells [105]. Furthermore, Zhang et al. demonstrated that CAFs-mediated HCC progression was associated with the loss of exosomal miR-320a derived from CAFs. By reprogramming with exogenous miR-320a, CAFs exerted miR-320a-mediated tumor-suppressing effects on HCC cells [106]. However, these strategies for controlling the evolvement of HCC are still limited and remain further pre-clinically investigated.

\section{Target paracrine productions of CAFs}

Aside from the strategies above, another promising strategy is to target CAF-derived cytokines and chemokines. Growing studies have suggested that the potentially targeted value of CAFs-secreted TGF- $\beta$ in the VM formation [57], tumor growth and invasion [46, 115]. Morén et al. indicated that liver $\mathrm{X}$ receptors $\alpha(\mathrm{LXR} \alpha)$ agonist T0901317 suppressed HCC growth by abrogating TGF- $\beta$ induced fibroblastic phenotypes of CAFs and hepatoma cells [42]. Mechanically, T0901317 repressed the transcriptional induction by TGF- $\beta$ stimulation through LXR $\alpha$ binding to the adjacent DNA motifs of the ACTA2 promoter. Indeed, several pharmacological approaches of impeding TGF- $\beta$ signaling have been developed to efficiently attenuate aggressiveness of HCC cells. It has been reported that TGF- $\beta$ signaling in fibroblasts inhibited $\mathrm{T}$ cells penetration and impaired the tumor response to anti-PD-L1 agent. Remarkably, co-administration of TGF- $\beta$-blocking and anti-PD-L1 antibodies provoked effective anti-tumor immunity and reversed the chemoresistance of anti-PD-L1 agent [116]. In addition, TGF- $\beta$ inhibitors have been developed and manifested with inhibitory effects on HCC cells. One typical representative TGF- $\beta$ receptor inhibitor LY2109761 interrupts the cross-talk between HCC cells and CAFs, leading to a significant reduction of $\mathrm{HCC}$ growth and dissemination [47]. More applausively, another TGF- $\beta$ inhibitor LY2157299 is evaluated at phase II clinical trial for HCC patients that fail in sorafenib treatment [117]. Recently, gold nanoparticles (GNPs) were found to alter cell morphology, migration, and molecular markers of CAFs by decreasing the levels of fibroblast activation protein TGF- $\beta 1$ [118].

Several chemokines containing CCL2/5/7 and CXCL16 were detected highly expressed in CAF-CM. Further investigation showed that these CAFs-derived chemokines facilitated HCC metastasis through activating Hh and TGF- $\beta$ pathways in HCC cells. The neutralizing antibodies of the chemokines obviously abolished CAFs-induced HCC migration, suggesting that CAFsgenerated chemokines are potential therapeutic targets for HCC [66]. Recent data demonstrated that CAFs released soluble CXCL12 into the HCC microenvironment and activated CXCL12/CXCR4/PI3K/AKT signaling of neighboring $\mathrm{HCC}$ cells, which subsequently alleviated apoptosis by elevating the BCL-2/BAX ratio. Indeed, downregulating CXCR4 abrogated the antiapoptotic effects triggered by CAFs, suggesting the underlying role of CXCL12/CXCR4 pathway in CAFsmediated apoptosis evasion in HCC milieu [49]. Previous studies have noted that the CXCL12-CXCR4 axis could induce $\mathrm{FAP}(+) \mathrm{CAFs}$-mediated immunosuppression by excluding CD8+ $\mathrm{T}$ cells. In a PDAC model, targeting the CXCL12-CXCR4 pathway by a specific CXCR4 inhibitor AMD3100 induced infiltration of CD8+ T cells into the tumors. Besides, CXCL12-induced $\mathrm{T}$ cell exclusion greatly hindered the anti-tumor effects of anti-PD-L1 monoclonal antibodies, thus AMD3100 synergized with $\alpha$-PD-L1 could greatly enhance the therapeutic effects on tumor cells [119]. However, the efficiency of targeting CXCL12-CXCR4 axis remain further evaluation in HCC.

Sun et al. indicated that Resolvin D1(RvD1), an endogenous anti-inflammatory lipid mediator, impeded CAFsinduced EMT and stemness features of HCC cells by suppressing the secretion of COMP [30]. RvD1 also impaired CAFs-derived COMP in a paracrine manner by targeting FPR2/ROS/FOXM1 signaling, ultimately blocking the FOXM1 recruitment to the COMP promoter. As 
one of the most abundant cytokines secreted by CAFs in HCC, IL-6 modulates immune response by regulating the generation of DCs, recruitment and function of neutrophils, and the induction of monocytes to differentiate into MDSCs. Recent studies showed that that blocking IL-6 enhanced antitumor immunity in HCC. IL-6 inhibitors could also reverse the anti-PD-L1 resistance of HCC. Moreover, synergized IL-6 and PD-L1 blockade effectively inhibited HCC growth in vivo. In a mice model of HCC, combinational treatment of IL- 6 blockade and anti-PD-L1 presented effectiveness with smaller tumor size and longer survival time [120]. In addition, Xiong et al. noted that IL- 6 neutralizing antibody depleted stem cell-like properties of HCC cells through inactivating STAT3/Notch signaling [65].

As is generally acknowledged that hepatic fibrosis greatly promotes occurrence of HCC, alleviating fibrosis may actually attenuate hepatocarcinogenesis. Accumulating clinical evidence have endorsed the efficiency of low-dose metronomic (LDM) chemotherapy regimen than traditional chemotherapy. Traditional maximumtolerated dose chemotherapy could unexpectedly induce an ELR+chemokine-producing phenotype in CAFs, whereby fostering chemoresistance and tumor progression. On the contrary, continuous LDM therapy largely downregulates therapeutics-induced CAFs paracrine signaling and prevents CAFs activation, resulting in an enhanced treatment response [121]. Currently, the effectiveness of either LDM chemotherapy alone or in combination with targeted therapeutics has been validated in several clinical trials [122]. Thus, it is a worthwhile potential approach to overcome CAFs-induced aggressive behaviors in HCC.

\section{Deplete CAFs by targeting surface markers}

$\alpha$-SMA is a typical marker of the myofibroblast subset of CAFs. However, the effects of targeting $\alpha$-SMA remains controversial on tumor progression. In a mouse model of breast cancer, targeting $\alpha-\mathrm{SMA}(+)$ CAFs obviously impeded tumor metastasis [123]. In contrast, blocking $\alpha$-SMA enhanced the infiltration of CD3+ Foxp3+ Tregs in tumors, thereby facilitating immunosuppressive TME for tumor progression [124]. Currently, the major strategy for depleting CAFs is mainly targeting another CAF surface marker FAP. Lang et al. constructed a CAF-targeting siRNA delivery system by loading the FAP- $\alpha$ antibody onto the cell-penetrating peptide-based nanoparticles, which specifically downregulated CXCL12 expression in CAFs [125]. Polyphyllin I could considerably inhibit FAP, SDF-1, and HGF in CAFs, and further suppress the growth of gastric cancer in vivo [126]. Genetic depletion of FAP reduced the infiltration of FAP $(+)$ CAFs with increased infiltration of $\mathrm{CD} 8+\mathrm{T}$ cells [127]. In addition, targeting FAP by DNA vaccines efficiently promoted $\mathrm{CD} 8+\mathrm{T}$ cell-mediated repression of CAFs, thereafter elevating intra-tumor chemotherapeutic drug uptake in multi-drug-resistant tumors [104]. To date, pre-clinical studies suggested targeting FAP $\alpha$ might be efficient approaches, including DNA vaccine, enzymatic inhibitor, neutralizing antibody, and chimeric antigen receptor T-cells [128]. Different from these strategies, Zhen et al. conducted a nanoparticle-based photoimmunotherapy that can selectively kill CAFs without causing systemic toxicity [129]. By using FAP-specific single chain variable fragment, this nano-approach efficiently eliminated CAFs and caused tumor suppression in tumor-bearing immunocompetent mice.

\section{Target precursors of CAFs}

Except for targeting CAF cell surface markers, alternative approach of targeting CAFs' precursors may efficiently reduce the generation of CAFs. Remarkably, as the main source of CAFs in HCC, the activated HSCs can be targeted by sibrotuzumab without toxic to normal hepatic cells [107]. Additionally, Chen et al. demonstrated that a genistein derivative DFOG intervened the crosstalk between HSCs and hepatic CSCs by downregulating FOXM1 expression and HGF secretion of HSCs. Metformin, a well-known anti-diabetes drug, was previously reported to prevent liver tumorigenesis by attenuating $\mathrm{HSCs}$ activation in $\mathrm{CCl} 4$ challenged transgenic mouse model [109]. Another widely used agent curcumin could significantly suppress the HSCs-induced aggressive behaviors of $\mathrm{HCC}$ cells by inhibiting reactive oxygen species (ROS)/HIF-1 $\alpha /$ CTGF signaling [110]. Currently, activated HSCs can also be inhibited by efficient antifibrotic drugs, including PRI-724, follistatin, Gliotoxin, salvianolic acid, sulfasalazine, Curcumin, tanshinone I, and conophylline [130-133]. It is worth noting that these drugs against liver fibrosis may also benefit for HCC treatment by blunting of CAFs' activation [94]. Apart from HSCs, PTFs might also be the candidate target. A pan-LPA inhibitor ( $\alpha$-bromomethylene phosphonateLPA) blocked the transformation from PTFs into CAFs, subsequently suppressing $\mathrm{HCC}$ growth and progression in vitro and in vivo [45].

\section{Block activation signaling and downstream effectors of CAFs}

Monocytic MDSCs (M-MDSCs) accumulated in fibrotic livers, which is associated with decreased tumor-infiltrating lymphocytes (TILs) and increased tumorigenicity in mouse models. In human HCC, M-MDSCs enriched in the para-cancerous fibrotic liver tissues are remarkably correlated with aggressive tumor phenotypes and shorter survival. Prior study indicated that M-MDSCs in HCC 
could be differentiate from monocytes activated by CAFs through monocyte-intrinsic p38 MAPK signaling. Interestingly, targeting the CAF-M-MDSC crosstalk using p38 MAPK inhibitor significantly enhanced the efficacy of anti-PD-L1 therapy and led to tumor eradication, ultimately prolonging survival in the fibrotic-HCC mouse model [111].

Almost half of the HCC cases show upregulated activity of mTOR pathway, which plays essential roles in tumor growth, proliferation and apoptosis. Since CAFs-derived factors trigger hepatocarcinogenesis partially through mTOR cascade signaling, inactivating mTOR signaling may be a potential treatment for HCC. Indeed, the mTOR signaling pathway inhibitor rapamycin exhibited antitumoral activity by interfering with progranulin or IL-6-mediated proliferation and invasion of HCC cells [62]. In lung cancer and melanoma, the IGF2 neutralizing antibody and the autophagy inhibitor 3-MA dramatically reduced the CAF-promoted tumor relapse in mice after radiotherapy by regulating $\mathrm{m}$-TOR-mediated autophagic activities. Additionally, mTOR pathway contributes to the synthesis of various secretions of $\alpha-S M A(+) C A F s$, thereby eliminating the CAF-mediated drug resistance in cancer cells. Therefore, targeting mTOR signaling attenuates the production of multifarious secretions, which provide an alternative option for cancer treatment [134]. As described above, BMP4 activated NFs into CAFs via SMAD signaling pathway. Further dorsomorphin (SMAD $1 / 5 / 8$ inhibitor) treatment suppressed the elevation of ACTA2 and IL-6 induced by exogenous BMP4 [51]. Aside from that, p-STAT3 (Tyr705) inhibitor cryptotanshinone could also deplete the CAFs-induced CSC effects on HCC cells by abrogating STAT3 signaling [65]. These evidences suggest that the key genes and signaling pathways involved in the activation of CAFs are promising molecular targets for HCC treatment.

\section{Conclusion}

Accumulating evidences suggest the pivotal roles of CAFs in favoring aggressive behaviors in HCC. In this review, we explicitly summarized the heterogeneity of CAFs in HCC, regarding cell origin, location, and phenotypes. Potential mechanisms by which CAFs fuel HCC progression include ECM remodeling, neovascularization, immunosuppression, EMT and stemness of HCC cells. Given the crucial roles in HCC progression, CAF may be an attractive target for the treatment of HCC. Growing studies have highlighted the benefits of "anti-CAFs" therapy for tumor patients. However, for the current status, it is formidable to target CAFs precisely without damaging normal tissue due to the elusive sources and less specific markers. Additionally, considering the existence of cancer-restraining CAFs in other cancer types, the exact roles of CAFs in HCC should be further determined [135, 136]. In conclusion, it is attached great significance to further investigating the roles and mechanisms involved in the CAFs in HCC progression, which will provide candidate targets for $\mathrm{HCC}$ treatment.

\section{Abbreviations}

HCC: Hepatocellular carcinoma; HCV: Hepatitis C virus; NAFLD: Non-alcoholic fatty liver disease; HBV: Chronic hepatitis B virus; NASH: Non-alcoholic

steatohepatitis; ALD: Alcohol-related liver disease; IR: Insulin resistance; HSCs: Hepatic stellate cells; ECM: Extracellular matrix; TKI: Tyrosine kinase inhibitor; OS: Over survival; PME: Premalignant microenvironment; TME: Tumor microenvironment; CAFs: Cancer-associated fibroblasts; TAMs: Tumor-associated macrophages; VM: Vasculogenic mimicry; ER: Endoplasmic reticulum; ED-A: Ectodysplasin-A; EGF: Epidermal growth factor; FGF: Fibroblast growth factor; HGF: Hypoxic growth factor; PDGF: Platelet derive growth factor; CXCL12: Chemokines like chemokine ligand 12; TGF- $\beta$ : Transforming growth factor- $\beta$; EndMT: Endothelial-to-mesenchymal transition; EMT: Epithelial-mesenchymal transition; a-SMA: a-Smooth muscle actin; FAP: Fibroblast activation protein; FSP1: Fibroblast specific protein 1; PDGFR: Platelet-derived growth factor receptor; PDAC: Pancreatic ductal adenocarcinoma; MSCs: Mesenchymal stromal cells; HSECs: Hepatic sinusoidal endothelial cells; PTFs: Peritumoral tissue fibroblasts; HIF-1a: Hypoxia inducible factor 1a; LPA: Lysophostatidic acid; PFs: Portal fibroblasts; LXR: Liver X receptors; CTGF: Connective tissue growth factor; CSCs: Cancer stem cells; TAFs: Tumor tissue-derived fibroblasts; COMP: Oligomeric matrix protein; VEGF: Vascular endothelial growth factor; PGF: Placental growth factor; MDSCs: Marrow-derived suppressor cells; Tregs: Regulatory T cells; MDSCs: Myeloid-derived suppressor cells; STAT3-PDL: Activate signal transducer and activator of Janus kinase-programmed cell death ligand 1; LDM: Low-dose metronomic; CM: Conditioned medium; EVs: Extracellular vesicles; DFS: Disease-free survival.

\section{Acknowledgements}

We thank Dr. Russel and Dr. Zhao for the English grammar revision of the manuscript.

\section{Authors' contributions}

$J Z, C G$ and QS contributed to drafting and editing of the manuscript, shared the first authorship. WZ and MX designed, revised and finalized the manuscript. MZ and YX contributed to literature search. All authors contributed to drafting and revising the article, gave final approval of the version to be published, and agree to be accountable for all aspects of the work. All authors read and approved the final manuscript.

\section{Funding}

This study was supported by grants from National Natural Science Foundation (81702419), the Key Research and Development Plan of Jiangsu Province (BE2019692), and Nantong Science and Technology Project (MS12019013, MS22018006)

\section{Availability of supporting data}

Not applicable.

Ethics approval and consent to participate

Not applicable.

\section{Consent for publication \\ Not applicable.}

\section{Competing interests}

The authors declare that they have no competing interests.

\section{Author details}

${ }^{1}$ Research Center of Clinical Medicine, Affiliated Hospital of Nantong University, 20 Xisi Road, Nantong 226001, Jiangsu, China. ${ }^{2}$ School of Medicine, Nantong University, 19 Qixiu Road, Nantong 226001, Jiangsu, China. ${ }^{3}$ Department of Radiology, Wake Forest School of Medicine, One Medical Center Boulevard, Winston-Salem, NC 27157, USA. 
Received: 10 June 2020 Accepted: 23 October 2020

Published online: 31 October 2020

\section{References}

1. Bray F, Ferlay J, Soerjomataram I, Siegel RL, Torre LA, Jemal A. Global cancer statistics 2018: GLOBOCAN estimates of incidence and mortality worldwide for 36 cancers in 185 countries. CA Cancer J Clin. 2018;68(6):394-424

2. El-Serag HB. Epidemiology of viral hepatitis and hepatocellular carcinoma. Gastroenterology. 2012;142(6):1264-1273.e1261.

3. Tang A, Hallouch O, Chernyak V, Kamaya A, Sirlin CB. Epidemiology of hepatocellular carcinoma: target population for surveillance and diagnosis. Abdom Radiol. 2018;43(1):13-25.

4. Medavaram S, Zhang Y. Emerging therapies in advanced hepatocellular carcinoma. Exp Hematol Oncol. 2018;7:17.

5. Altekruse SF, McGlynn KA, Reichman ME. Hepatocellular carcinoma incidence, mortality, and survival trends in the United States from 1975 to 2005. J Clin Oncol. 2009;27(9):1485-91.

6. Hajarizadeh B, Grebely J, Dore GJ. Epidemiology and natural history of HCV infection. Nat Rev Gastroenterol Hepatol. 2013;10(9):553-62.

7. Farrell GC, Wong VW, Chitturi S. NAFLD in Asia-as common and important as in the West. Nat Rev Gastroenterol Hepatol. 2013;10(5):307-18.

8. El-Serag HB. Hepatocellular carcinoma. N Engl J Med. 2011;365(12):1118-27.

9. Llovet JM, Ricci S, Mazzaferro V, Hilgard P, Gane E, Blanc JF, et al. Sorafenib in advanced hepatocellular carcinoma. N Engl J Med. 2008:359(4):378-90.

10. Kudo M, Finn RS, Qin S, Han KH, Ikeda K, Piscaglia F, et al. Lenvatinib versus sorafenib in first-line treatment of patients with unresectable hepatocellular carcinoma: a randomised phase 3 non-inferiority trial. Lancet. 2018;391(10126):1163-73.

11. Shlomai A, Leshno M, Goldstein DA. Regorafenib treatment for patients with hepatocellular carcinoma who progressed on sorafenib-a costeffectiveness analysis. PLoS ONE. 2018;13(11):e0207132.

12. Abou-Alfa GK, Meyer T, Cheng AL, El-Khoueiry AB, Rimassa L, Ryoo BY, et al. Cabozantinib in patients with advanced and progressing hepatocellular carcinoma. N Engl J Med. 2018;379(1):54-63.

13. El-Khoueiry AB, Sangro B, Yau T, Crocenzi TS, Kudo M, Hsu C, et al. Nivolumab in patients with advanced hepatocellular carcinoma (CheckMate 040): an open-label, non-comparative, phase 1/2 dose escalation and expansion trial. Lancet. 2017;389(10088):2492-502.

14. Zhu AX, Finn RS, Edeline J, Cattan S, Ogasawara S, Palmer D, et al. Pembrolizumab in patients with advanced hepatocellular carcinoma previously treated with sorafenib (KEYNOTE-224): a non-randomised, open-label phase 2 trial. Lancet Oncol. 2018;19(7):940-52.

15. Affo $S, Y u L X$, Schwabe RF. The role of cancer-associated fibroblasts and fibrosis in liver cancer. Annu Rev Pathol. 2017;12:153-86.

16. EbioMedicine. The tumor microenvironment: a druggable target for metastatic disease? EBioMedicine 2018:31:1-2

17. Baglieri J, Brenner DA, Kisseleva T. The role of fibrosis and liver-associated fibroblasts in the pathogenesis of hepatocellular carcinoma. Int J Mol Sci. 2019;20(7):1723

18. Hernandez-Gea V, Toffanin S, Friedman SL, Llovet JM. Role of the microenvironment in the pathogenesis and treatment of hepatocellular carcinoma. Gastroenterology. 2013;144(3):512-27.

19. Yin Z, Jiang K, Li R, Dong C, Wang L. Multipotent mesenchymal stromal cells play critical roles in hepatocellular carcinoma initiation, progression and therapy. Mol Cancer. 2018;17(1):178.

20. Yoshida K, Takanashi A, Kyo E, Ito M, Ito H, Niimoto M, et al. Epidermal growth factor induces the expression of its receptor gene in human gastric carcinoma cell line TMK-1. Jpn J Cancer Res. 1989;80(8):743-6.

21. Khan GJ, Sun L, Khan S, Yuan S, Nongyue H. Versatility of cancer associated fibroblasts: commendable targets for anti-tumor therapy. Curr Drug Targets. 2018;19(13):1573-88.

22. Prakash J. Cancer-associated fibroblasts: perspectives in cancer therapy. Trends Cancer. 2016:2(6):277-9.

23. Ishii G, Ochiai A, Neri S. Phenotypic and functional heterogeneity of cancer-associated fibroblast within the tumor microenvironment. Adv Drug Deliv Rev. 2016;99(Pt B):186-96.
24. Ohlund D, Handly-Santana A, Biffi G, Elyada E, Almeida AS, Ponz-Sarvise $M$, et al. Distinct populations of inflammatory fibroblasts and myofibroblasts in pancreatic cancer. J Exp Med. 2017:214(3):579-96.

25. Lynch MD, Watt FM. Fibroblast heterogeneity: implications for human disease. J Clin Investig. 2018;128(1):26-35

26. Arnold JN, Magiera L, Kraman M, Fearon DT. Tumoral immune suppression by macrophages expressing fibroblast activation protein-alpha and heme oxygenase-1. Cancer Immunol Res. 2014;2(2):121-6.

27. Costa A, Kieffer Y, Scholer-Dahirel A, Pelon F, Bourachot B, Cardon M, et al. Fibroblast heterogeneity and immunosuppressive environment in human breast cancer. Cancer Cell. 2018;33(3):463-479.e410.

28. Neuzillet C, Tijeras-Raballand A, Ragulan C, Cros J, Patil Y, Martinet M, et al. Inter- and intra-tumoural heterogeneity in cancer-associated fibroblasts of human pancreatic ductal adenocarcinoma. J Pathol. 2019;248(1):51-65.

29. Krenkel O, Hundertmark J, Ritz TP, Weiskirchen R, Tacke F. Single cell RNA sequencing identifies subsets of hepatic stellate cells and myofibroblasts in liver fibrosis. Cells. 2019;8(5):503.

30. Sun L, Wang Y, Wang L, Yao B, Chen T, Li Q, et al. Resolvin D1 prevents epithelial-mesenchymal transition and reduces the stemness features of hepatocellular carcinoma by inhibiting paracrine of cancer-associated fibroblast-derived COMP. J Exp Clin Cancer Res. 2019;38(1):170.

31. Nayar S, Campos J, Smith CG, lannizzotto V, Gardner DH, Mourcin F, et al Immunofibroblasts are pivotal drivers of tertiary lymphoid structure formation and local pathology. Proc Natl Acad Sci. 2019;116(27):13490-7.

32. Lambrechts D, Wauters E, Boeckx B, Aibar S, Nittner D, Burton O, et al. Phenotype molding of stromal cells in the lung tumor microenvironment. Nat Med. 2018;24(8):1277-89.

33. Henderson NC, Arnold TD, Katamura Y, Giacomini MM, Rodriguez JD, McCarty JH, et al. Targeting of alphav integrin identifies a core molecular pathway that regulates fibrosis in several organs. Nat Med. 2013;19(12):1617-24.

34. Mederacke I, Hsu CC, Troeger JS, Huebener P, Mu X, Dapito DH, et al. Fate tracing reveals hepatic stellate cells as dominant contributors to liver fibrosis independent of its aetiology. Nat Commun. 2013;4:2823.

35. Puche JE, Lee YA, Jiao J, Aloman C, Fiel MI, Munoz U, et al. A novel murine model to deplete hepatic stellate cells uncovers their role in amplifying liver damage in mice. Hepatology. 2013;57(1):339-50.

36. Bataller R, Brenner DA. Liver fibrosis. J Clin Investig. 2005;115(2):209-18.

37. Shimizu S, Yamada N, Sawada T, Ikeda K, Kawada N, Seki S, et al. In vivo and in vitro interactions between human colon carcinoma cells and hepatic stellate cells. Jpn J Cancer Res. 2000;91(12):1285-95.

38. Tan HX, Gong WZ, Zhou K, Xiao ZG, Hou FT, Huang T, et al. CXCR4/TGFbeta 1 mediated hepatic stellate cells differentiation into carcinomaassociated fibroblasts and promoted liver metastasis of colon cancer. Cancer Biol Ther. 2020;21(3):258-68.

39. Wright JH, Johnson MM, Shimizu-Albergine M, Bauer RL, Hayes BJ, Surapisitchat J, et al. Paracrine activation of hepatic stellate cells in platelet-derived growth factor $\mathrm{C}$ transgenic mice: evidence for stromal induction of hepatocellular carcinoma. Int J Cancer. 2014;134(4):778-88.

40. Zhou Y, Ren H, Dai B, Li J, Shang L, Huang J, et al. Hepatocellular carcinoma-derived exosomal miRNA-21 contributes to tumor progression by converting hepatocyte stellate cells to cancer-associated fibroblasts. J Exp Clin Cancer Res. 2018;37(1):324.

41. Zou B, Liu X, Zhang B, Gong Y, Cai C, Li P, et al. The expression of FAP in hepatocellular carcinoma cells is induced by hypoxia and correlates with poor clinical outcomes. J Cancer. 2018;9(18):3278-86.

42. Moren A, Bellomo C, Tsubakihara Y, Kardassis D, Mikulits W, Heldin CH, et al. LXRalpha limits TGFbeta-dependent hepatocellular carcinoma associated fibroblast differentiation. Oncogenesis. 2019;8(6):36

43. Bhattacharya SD, Mi Z, Talbot LJ, Guo H, Kuo PC. Human mesenchymal stem cell and epithelial hepatic carcinoma cell lines in admixture: concurrent stimulation of cancer-associated fibroblasts and epithelialto-mesenchymal transition markers. Surgery. 2012;152(3):449-54.

44. Yeon JH, Jeong HE, Seo H, Cho S, Kim K, Na D, et al. Cancer-derived exosomes trigger endothelial to mesenchymal transition followed by the induction of cancer-associated fibroblasts. Acta Biomater. 2018;76:146-53.

45. Mazzocca A, Dituri F, Lupo L, Quaranta M, Antonaci S, Giannelli G. Tumor-secreted lysophostatidic acid accelerates hepatocellular 
carcinoma progression by promoting differentiation of peritumoral fibroblasts in myofibroblasts. Hepatology. 2011;54(3):920-30.

46. Giannelli G, Villa E, Lahn M. Transforming growth factor-beta as a therapeutic target in hepatocellular carcinoma. Cancer Res. 2014;74(7):1890-4.

47. Mazzocca A, Fransvea E, Dituri F, Lupo L, Antonaci S, Giannelli G. Down-regulation of connective tissue growth factor by inhibition of transforming growth factor beta blocks the tumor-stroma cross-talk and tumor progression in hepatocellular carcinoma. Hepatology. 2010;51(2):523-34.

48. Zhang R, Gao X, Zuo J, Hu B, Yang J, Zhao J, et al. STMN1 upregulation mediates hepatocellular carcinoma and hepatic stellate cell crosstalk to aggravate cancer by triggering the MET pathway. Cancer Sci. 2020;111(2):406-17.

49. Song T, Dou C, Jia Y, Tu K, Zheng X. TIMP-1 activated carcinomaassociated fibroblasts inhibit tumor apoptosis by activating SDF1/CXCR4 signaling in hepatocellular carcinoma. Oncotarget. 2015;6(14):12061-79.

50. Huang TX, Guan XY, Fu L. Therapeutic targeting of the crosstalk between cancer-associated fibroblasts and cancer stem cells. Am J Cancer Res. 2019;9(9):1889-904.

51. Mano Y, Yoshio S, Shoji H, Tomonari S, Aoki Y, Aoyanagi N, et al. Bone morphogenetic protein 4 provides cancer-supportive phenotypes to liver fibroblasts in patients with hepatocellular carcinoma. J Gastroenterol. 2019;54(11):1007-18

52. Labernadie A, Kato T, Brugués A, Serra-Picamal X, Derzsi S, Arwert E, et al. A mechanically active heterotypic E-cadherin/N-cadherin adhesion enables fibroblasts to drive cancer cell invasion. Nat Cell Biol. 2017;19(3):224-37.

53. Park SY, Jeong KJ, Panupinthu N, Yu S, Lee J, Han JW, et al. Lysophosphatidic acid augments human hepatocellular carcinoma cell invasion through LPA1 receptor and MMP-9 expression. Oncogene. 2011;30(11):1351-9.

54. Jia CC, Wang TT, Liu W, Fu BS, Hua X, Wang GY, et al. Cancer-associated fibroblasts from hepatocellular carcinoma promote malignant cell proliferation by HGF secretion. PLoS ONE. 2013;8(5):e63243.

55. Lau EY, Lo J, Cheng BY, Ma MK, Lee JM, Ng JK, et al. Cancer-associated fibroblasts regulate tumor-initiating cell plasticity in hepatocelIular carcinoma through c-Met/FRA1/HEY1 signaling. Cell Rep. 2016:15(6):1175-89.

56. Li Y, Wang R, Xiong S, Wang X, Zhao Z, Bai S, et al. Cancer-associated fibroblasts promote the stemness of CD24(+) liver cells via paracrine signaling. J Mol Med. 2019;97(2):243-55.

57. Yang J, Lu Y, Lin $Y Y$, Zheng $Z Y$, Fang JH, He S, et al. Vascular mimicry formation is promoted by paracrine TGF-beta and SDF1 of cancer-associated fibroblasts and inhibited by miR-101 in hepatocellular carcinoma. Cancer Lett. 2016:383(1):18-27.

58. Corpechot C, Barbu V, Wendum D, Kinnman N, Rey C, Poupon R, et al. Hypoxia-induced VEGF and collagen I expressions are associated with angiogenesis and fibrogenesis in experimental cirrhosis. Hepatology. 2002;35(5):1010-21.

59. Crawford Y, Kasman I, Yu L, Zhong C, Wu X, Modrusan Z, et al. PDGF-C mediates the angiogenic and tumorigenic properties of fibroblasts associated with tumors refractory to anti-VEGF treatment. Cancer Cell. 2009;15(1):21-34.

60. Liu Z, Chen M, Zhao R, Huang Y, Liu F, Li B, et al. CAF-induced placental growth factor facilitates neoangiogenesis in hepatocellular carcinoma. Acta Biochim Biophys Sin. 2020;52(1):18-25.

61. Deng Y, Cheng J, Fu B, Liu W, Chen G, Zhang Q, et al. Hepatic carcinomaassociated fibroblasts enhance immune suppression by facilitating the generation of myeloid-derived suppressor cells. Oncogene. 2017;36(8):1090-101.

62. Liu F, Zhang W, Yang F, Feng T, Zhou M, Yu Y, et al. Interleukin-6-stimulated progranulin expression contributes to the malignancy of hepatocellular carcinoma cells by activating mTOR signaling. Sci Rep. 2016:6:21260

63. Cheng JT, Deng YN, Yi HM, Wang GY, Fu BS, Chen WJ, et al. Hepatic carcinoma-associated fibroblasts induce IDO-producing regulatory dendritic cells through IL-6-mediated STAT3 activation. Oncogenesis. 2016:5:e198.
64. Cheng Y, Li H, Deng Y, Tai Y, Zeng K, Zhang Y, et al. Cancer-associated fibroblasts induce PDL1+ neutrophils through the IL6-STAT3 pathway that foster immune suppression in hepatocellular carcinoma. Cell Death Dis. 2018;9(4):422.

65. Xiong S, Wang R, Chen Q, Luo J, Wang J, Zhao Z, et al. Cancer-associated fibroblasts promote stem cell-like properties of hepatocellular carcinoma cells through IL-6/STAT3/Notch signaling. Am J Cancer Res. 2018;8(2):302-16

66. Liu J, Chen S, Wang W, Ning BF, Chen F, Shen W, et al. Cancer-associated fibroblasts promote hepatocellular carcinoma metastasis through chemokine-activated hedgehog and TGF-beta pathways. Cancer Lett. 2016;379(1):49-59.

67. Luo $Q$, Wang $C Q$, Yang $L Y$, Gao XM, Sun $H T$, Zhang Y, et al. FOXQ1/ NDRG1 axis exacerbates hepatocellular carcinoma initiation via enhancing crosstalk between fibroblasts and tumor cells. Cancer Lett. 2018:417:21-34.

68. Han SY, Han HB, Tian XY, Sun H, Xue D, Zhao C, et al. MicroRNA-33a-3p suppresses cell migration and invasion by directly targeting PBX3 in human hepatocellular carcinoma. Oncotarget. 2016;7(27):42461-73.

69. Malandrino A, Mak M, Kamm RD, Moeendarbary E. Complex mechanics of the heterogeneous extracellular matrix in cancer. Extreme Mech Lett. 2018:21:25-34.

70. Levental KR, Yu H, Kass L, Lakins JN, Egeblad M, Erler JT, et al. Matrix crosslinking forces tumor progression by enhancing integrin signaling. Cell. 2009;139(5):891-906

71. Attieh Y, Vignjevic DM. The hallmarks of CAFs in cancer invasion. Eur J Cell Biol. 2016;95(11):493-502.

72. Santamato A, Fransvea E, Dituri F, Caligiuri A, Quaranta M, Niimi T, et al. Hepatic stellate cells stimulate HCC cell migration via laminin-5 production. Clin Sci. 2011;121(4):159-68.

73. Schrader J, Gordon-Walker TT, Aucott RL, van Deemter M, Quaas A, Walsh S, et al. Matrix stiffness modulates proliferation, chemotherapeutic response, and dormancy in hepatocellular carcinoma cells. Hepatology. 2011;53(4):1192-205.

74. Yamashita T, Wang XW. Cancer stem cells in the development of liver cancer. J Clin Investig. 2013;123(5):1911-8.

75. Flores-Tellez TN, Villa-Trevino S, Pina-Vazquez C. Road to stemness in hepatocellular carcinoma. World J Gastroenterol. 2017;23(37):6750-76.

76. Jiang J, Ye F, Yang X, Zong C, Gao L, Yang Y, et al. Peri-tumor associated fibroblasts promote intrahepatic metastasis of hepatocellular carcinoma by recruiting cancer stem cells. Cancer Lett. 2017;404:19-28.

77. Zhao Z, Bai S, Wang R, Xiong S, Li Y, Wang X, et al. Cancer-associated fibroblasts endow stem-like qualities to liver cancer cells by modulating autophagy. Cancer Manag Res. 2019;11:5737-44.

78. Liu C, Liu L, Chen X, Cheng J, Zhang H, Zhang C, et al. LSD1 stimulates cancer-associated fibroblasts to drive Notch3-dependent self-renewal of liver cancer stem-like cells. Cancer Res. 2018;78(4):938-49.

79. Peng H, Xue R, Ju Z, Qiu J, Wang J, Yan W, et al. Cancer-associated fibroblasts enhance the chemoresistance of CD73(+) hepatocellular carcinoma cancer cells via HGF-Met-ERK1/2 pathway. Ann TransI Med. 2020;8(14):856.

80. Khawar IA, Park JK, Jung ES, Lee MA, Chang S, Kuh HJ. Three dimensional mixed-cell spheroids mimic stroma-mediated chemoresistance and invasive migration in hepatocellular carcinoma. Neoplasia. 2018;20(8):800-12.

81. Liu J, Li P, Wang L, Li M, Ge Z, Noordam L, et al. Cancer-associated fibroblasts provide a stromal niche for liver cancer organoids that confers trophic effects and therapy resistance. Cell Mol Gastroenterol Hepatol. 2020. https://doi.org/10.1016/j.jcmgh.2020.09.003.

82. Aronovich A, Moyal L, Gorovitz B, Amitay-Laish I, Naveh HP, Forer Y, et al. Cancer-associated fibroblasts in mycosis fungoides promote tumor cell migration and drug resistance via CXCL12/CXCR4. J Investig Dermatol. 2020. https://doi.org/10.1016/j.jid.2020.06.034.

83. Zhang F, Cui JY, Gao HF, Yu H, Gao FF, Chen JL, et al. Cancer-associated fibroblasts induce epithelial-mesenchymal transition and cisplatin resistance in ovarian cancer via CXCL12/CXCR4 axis. Future Oncol. 2020. https://doi.org/10.2217/fon-2020-0095.

84. Zhang Z, Karthaus WR, Lee YS, Gao VR, Wu C, Russo JW, et al. Tumor microenvironment-derived NRG1 promotes antiandrogen resistance in prostate cancer. Cancer Cell. 2020;38(2):279-96. 
85. Chi CW, Lao YH, Ahmed AHR, Benoy EC, Li C, Dereli-Korkut Z, et al. Highthroughput tumor-on-a-chip platform to study tumor-stroma interactions and drug pharmacokinetics. Adv Healthc Mater. 2020:e2000880.

86. Uchihara T, Miyake K, Yonemura A, Komohara Y, Itoyama R, Koiwa M, et al. Extracellular vesicles from cancer-associated fibroblasts containing annexin A6 induces FAK-YAP activation by stabilizing beta1 integrin. Enhanc Drug Resist Cancer Res. 2020;80(16):3222-35.

87. Kubo N, Araki K, Kuwano H, Shirabe K. Cancer-associated fibroblasts in hepatocellular carcinoma. World J Gastroenterol. 2016:22(30):6841-50.

88. Ho MC, Chen CN, Lee H, Hsieh FJ, Shun CT, Chang CL, et al. Placenta growth factor not vascular endothelial growth factor $\mathrm{A}$ or $\mathrm{C}$ can predict the early recurrence after radical resection of hepatocellular carcinoma. Cancer Lett. 2007:250(2):237-49.

89. Xu HX, Zhu XD, Zhuang PY, Zhang JB, Zhang W, Kong LQ, et al. Expression and prognostic significance of placental growth factor in hepatocellular carcinoma and peritumoral liver tissue. Int J Cancer. 2011;128(7):1559-69.

90. Huang B, Huang M, Li Q. Cancer-associated fibroblasts promote angiogenesis of hepatocellular carcinoma by VEGF-mediated EZH2NASH1 pathway. Technol Cancer Res Treat. 2019;18:1533033819879905.

91. Folberg R, Hendrix MJ, Maniotis AJ. Vasculogenic mimicry and tumor angiogenesis. Am J Pathol. 2000;156(2):361-81.

92. Liu B, Salgado OC, Singh S, Hippen KL, Maynard JC, Burlingame AL, et al. The lineage stability and suppressive program of regulatory $T$ cells require protein O-GIcNAcylation. Nat Commun. 2019;10(1):354.

93. Jiang X, Wang J, Deng X, Xiong F, Ge J, Xiang B, et al. Role of the tumor microenvironment in PD-L1/PD-1-mediated tumor immune escape. Mol Cancer. 2019;18(1):10.

94. Lu C, Rong D, Zhang B, Zheng W, Wang X, Chen Z, et al. Current perspectives on the immunosuppressive tumor microenvironment in hepatocellular carcinoma: challenges and opportunities. Mol Cancer. 2019;18(1):130.

95. Monteran L, Erez N. The dark side of fibroblasts: cancer-associated fibroblasts as mediators of immunosuppression in the tumor microenvironment. Front Immunol. 2019;10:1835.

96. Yang X, Lin Y, Shi Y, Li B, Liu W, Yin W, et al. FAP promotes immunosuppression by cancer-associated fibroblasts in the tumor microenvironment via STAT3-CCL2 signaling. Cancer Res. 2016;76(14):4124-35.

97. Li T, Yang Y, Hua X, Wang G, Liu W, Jia C, et al. Hepatocellular carcinomaassociated fibroblasts trigger NK cell dysfunction via PGE2 and IDO. Cancer Lett. 2012;318(2):154-61.

98. Fang M, Yuan J, Chen M, Sun Z, Liu L, Cheng G, et al. The heterogenic tumor microenvironment of hepatocellular carcinoma and prognostic analysis based on tumor neo-vessels, macrophages and alpha-SMA. Oncol Lett. 2018;15(4):4805-12.

99. Takamura H, Nakanuma S, Hayashi H, Tajima H, Kakinoki K, Sakai S, et al. Evaluation of eligibility criteria in living donor liver transplantation for hepatocellular carcinoma by alpha-SMA-positive cancer-associated fibroblasts. Oncol Rep. 2013;30(4):1561-74.

100. Yang XY, Zhang D, Zou QF, Fan F, Shen F. Association of tumor-associated fibroblasts with progression of hepatocellular carcinoma. Med Oncol. 2013;30(3):593.

101. Zhao RC, Zhou J, Chen KF, Gong J, Liu J, He JY, et al. The prognostic value of combination of CD90 and OCT4 for hepatocellular carcinoma after curative resection. Neoplasma. 2016;63(2):288-98.

102. Liu Z, Chen M, Zhao R, Huang Y, Liu F, Li B, et al. CAF-induced placental growth factor facilitates neoangiogenesis in hepatocellular carcinoma. Acta Biochim Biophys Sin. 2020;52(1):18-25.

103. Ma L, Wang XY, Duan M, Liu LZ, Shi JY, Dong LQ, et al. Telomere length variation in tumor cells and cancer-associated fibroblasts: potential biomarker for hepatocellular carcinoma. J Pathol. 2017;243(4):407-17.

104. Loeffler M, Kruger JA, Niethammer AG, Reisfeld RA. Targeting tumorassociated fibroblasts improves cancer chemotherapy by increasing intratumoral drug uptake. J Clin Investig. 2006;116(7):1955-62.

105. Wang F, Li L, Piontek K, Sakaguchi M, Selaru FM. Exosome miR-335 as a novel therapeutic strategy in hepatocellular carcinoma. Hepatology. 2018;67(3):940-54.

106. Zhang Z, Li X, Sun W, Yue S, Yang J, Li J, et al. Loss of exosomal miR-320a from cancer-associated fibroblasts contributes to HCC proliferation and metastasis. Cancer Lett. 2017;397:33-42.
107. Kelly T. Fibroblast activation protein-alpha and dipeptidyl peptidase IV (CD26): cell-surface proteases that activate cell signaling and are potential targets for cancer therapy. Drug Resist Updat. 2005;8(1-2):51-8.

108. Chen A, Xu C, Luo Y, Liu L, Song K, Deng G, et al. Disruption of crosstalk between $L X-2$ and liver cancer stem-like cells from MHCC97H cells by DFOG via inhibiting FOXM1. Acta Biochim Biophys Sin. 2019;51(12):1267-75.

109. Shankaraiah RC, Callegari E, Guerriero P, Rimessi A, Pinton P, Gramantieri $L$, et al. Metformin prevents liver tumourigenesis by attenuating fibrosis in a transgenic mouse model of hepatocellular carcinoma. Oncogene. 2019;38(45):7035-45.

110. Shao S, Duan W, Xu Q, Li X, Han L, Li W, et al. Curcumin suppresses hepatic stellate cell-induced hepatocarcinoma angiogenesis and invasion through downregulating CTGF. Oxid Med Cell Longev. 2019;2019:8148510.

111. Liu M, Zhou J, Liu X, Feng Y, Yang W, Wu F, et al. Targeting monocyteintrinsic enhancer reprogramming improves immunotherapy efficacy in hepatocellular carcinoma. Gut. 2020;69(2):365-79.

112. Kisseleva T, Cong M, Paik Y, Scholten D, Jiang C, Benner C, et al. Myofibroblasts revert to an inactive phenotype during regression of liver fibrosis. Proc Natl Acad Sci USA. 2012;109(24):9448-53.

113. Ernsting MJ, Hoang B, Lohse I, Undzys E, Cao P, Do T, et al. Targeting of metastasis-promoting tumor-associated fibroblasts and modulation of pancreatic tumor-associated stroma with a carboxymethylcellulosedocetaxel nanoparticle. J Control Release. 2015;206:122-30.

114. Miao L, Liu Q, Lin CM, Luo C, Wang Y, Liu L, et al. Targeting tumor-associated fibroblasts for therapeutic delivery in desmoplastic tumors. Cancer Res. 2017;77(3):719-31.

115. Farazi PA, DePinho RA. Hepatocellular carcinoma pathogenesis: from genes to environment. Nat Rev Cancer. 2006;6(9):674-87.

116. Mariathasan S, Turley SJ, Nickles D, Castiglioni A, Yuen K, Wang Y, et al. TGFbeta attenuates tumour response to PD-L1 blockade by contributing to exclusion of T cells. Nature. 2018:554(7693):544-8.

117. Herbertz S, Sawyer JS, Stauber AJ, Gueorguieva I, Driscoll KE, Estrem ST, et al. Clinical development of galunisertib (LY2157299 monohydrate), a small molecule inhibitor of transforming growth factor-beta signaling pathway. Drug Des Dev Ther. 2015;9:4479-99.

118. Zhang Y, Elechalawar CK, Hossen MN, Francek ER, Dey A, Wilhelm $\mathrm{S}$, et al. Gold nanoparticles inhibit activation of cancer-associated fibroblasts by disrupting communication from tumor and microenvironmental cells. Bioact Mater. 2021;6(2):326-32.

119. Feig C, Jones JO, Kraman M, Wells RJ, Deonarine A, Chan DS, et al. Targeting CXCL12 from FAP-expressing carcinoma-associated fibroblasts synergizes with anti-PD-L1 immunotherapy in pancreatic cancer. Proc Natl Acad Sci U S A. 2013;110(50):20212-7.

120. Liu H, Shen J, Lu K. IL-6 and PD-L1 blockade combination inhibits hepatocellular carcinoma cancer development in mouse model. Biochem Biophys Res Commun. 2017;486(2):239-44.

121. Chan TS, Hsu CC, Pai VC, Liao WY, Huang SS, Tan KT, et al. Metronomic chemotherapy prevents therapy-induced stromal activation and induction of tumor-initiating cells. J Exp Med. 2016;213(13):2967-88.

122. Kerbel RS, Grothey A. Gastrointestinal cancer: Rationale for metronomic chemotherapy in phase III trials. Nat Rev Clin Oncol. 2015;12(6):313-4.

123. Murakami M, Ernsting MJ, Undzys E, Holwell N, Foltz WD, Li SD. Docetaxel conjugate nanoparticles that target alpha-smooth muscle actinexpressing stromal cells suppress breast cancer metastasis. Cancer Res. 2013;73(15):4862-71.

124. Ozdemir BC, Pentcheva-Hoang T, Carstens JL, Zheng X, Wu CC, Simpson $\mathrm{TR}$, et al. Depletion of carcinoma-associated fibroblasts and fibrosis induces immunosuppression and accelerates pancreas cancer with reduced survival. Cancer Cell. 2014;25(6):719-34.

125. Lang J, Zhao X, Qi Y, Zhang Y, Han X, Ding Y, et al. Reshaping prostate tumor microenvironment to suppress metastasis via cancer-associated fibroblast inactivation with peptide-assembly-based nanosystem. ACS Nano. 2019;13(11):12357-71.

126. Dong R, Guo J, Zhang Z, Zhou Y, Hua Y. Polyphyllin I inhibits gastric cancer cell proliferation by downregulating the expression of fibroblast activation protein alpha (FAP) and hepatocyte growth factor (HGF) in cancer-associated fibroblasts. Biochem Biophys Res Commun. 2018:497(4):1129-34. 
127. Kraman M, Bambrough PJ, Arnold JN, Roberts EW, Magiera L, Jones JO, et al. Suppression of antitumor immunity by stromal cells expressing fibroblast activation protein-alpha. Science. 2010;330(6005):827-30.

128. Hofheinz RD, Weisser A, Willer A, Hehlmann R, Hochhaus A. Treatment of a patient with advanced esophageal cancer with a combination of mitomycin C and capecitabine: activation of the thymidine phosphorylase as active principle? Onkologie. 2003;26(2):161-4.

129. Zhen Z, Tang W, Wang M, Zhou S, Wang H, Wu Z, et al. Protein nanocage mediated fibroblast-activation protein targeted photoimmunotherapy to enhance cytotoxic T cell infiltration and tumor control. Nano Lett. 2017;17(2):862-9.

130. Osawa Y, Oboki K, Imamura J, Kojika E, Hayashi Y, Hishima T, et al. Inhibition of cyclic adenosine monophosphate (CAMP)-response elementbinding protein (CREB)-binding protein (CBP)/beta-catenin reduces liver fibrosis in mice. EBioMedicine. 2015;2(11):1751-8.

131. Kubo N, Saito R, Hamano K, Nagasawa M, Aoki F, Takei I, et al. Conophylline suppresses hepatic stellate cells and attenuates thioacetamideinduced liver fibrosis in rats. Liver Int. 2014:34(7):1057-67.

132. Wright MC, Issa R, Smart DE, Trim N, Murray Gl, Primrose JN, et al. Gliotoxin stimulates the apoptosis of human and rat hepatic stellate cells and enhances the resolution of liver fibrosis in rats. Gastroenterology. 2001;121(3):685-98.

133. Tsai MK, Lin YL, Huang YT. Effects of salvianolic acids on oxidative stress and hepatic fibrosis in rats. Toxicol Appl Pharmacol. 2010;242(2):155-64.

134. Duluc C, Moatassim-Billah S, Chalabi-Dchar M, Perraud A, Samain R, Breibach F, et al. Pharmacological targeting of the protein synthesis mTOR/4E-BP1 pathway in cancer-associated fibroblasts abrogates pancreatic tumour chemoresistance. EMBO Mol Med. 2015;7(6):735-53.

135. Kobayashi H, Enomoto A, Woods SL, Burt AD, Takahashi M, Worthley DL. Cancer-associated fibroblasts in gastrointestinal cancer. Nat Rev Gastroenterol Hepatol. 2019;16(5):282-95.

136. Miyai Y, Esaki N, Takahashi M, Enomoto A. Cancer-associated fibroblasts that restrain cancer progression: hypotheses and perspectives. Cancer Sci. 2020;111(4):1047-57.

\section{Publisher's Note}

Springer Nature remains neutral with regard to jurisdictional claims in published maps and institutional affiliations.
Ready to submit your research? Choose BMC and benefit from:

- fast, convenient online submission

- thorough peer review by experienced researchers in your field

- rapid publication on acceptance

- support for research data, including large and complex data types

- gold Open Access which fosters wider collaboration and increased citations

- maximum visibility for your research: over $100 \mathrm{M}$ website views per year

At BMC, research is always in progress.

Learn more biomedcentral.com/submissions 